\title{
VIABILIDAD TECNOLÓGICA DE LA INSTALACIÓN DE UNA PLANTA PRODUCTORA DE UNA BEBIDA A PARTIR DE ALGARROBO (PROSOPIS PALLIDA), TARWI (LUPINUS MUTABILIS) Y QUINUA (CHenopodium QUinoa) en EL Perú
}

\author{
Maggie Lalesca ManRique Pasión* \\ https://orcid.org/0000-0001-9826-1695 \\ Rocío del Pilar Valverde Moreno* \\ https://orcid.org/0000-0003-0679-2988 \\ RAFAel VilLanueva Flores* \\ https://orcid.org/0000-0003-1056-251X \\ Universidad de Lima, Perú
}

Recibido: 13 de octubre del 2020 / Aprobado: 19 de mayo del 2021

doi: https://doi.org/10.26439/ing.ind2021.n41.5545

RESUMEN: El presente artículo tiene como objetivo exponer la viabilidad tecnológica de la instalación de una planta productora de una bebida a base de algarrobo, tarwi y quinua a través de la definición del producto, del proceso de producción y de la capacidad de la planta. Esta bebida es una alternativa a la gran cantidad de jugos y gaseosas que tienen un alto contenido de azúcar. Además, dado a que está hecha a base de una mezcla de dos legumbres y un pseudocereal, aporta aminoácidos esenciales al organismo.

PALABRAS CLAVE: tarwi / quinua / algarrobo / bebida / viabilidad tecnológica / capacidad de planta

\section{THE TECHNOLOGICAL FEASIBILITY OF THE INSTALLATION OF A PRODUCTION PLANT FOR A DRINK FROM CAROB (PROSOPIS PALLIDA), TARWI (LUPINUS MUTABILIS), AND QUINOA (CHENOPODIUM QUINOA)}

ABSTRACT: This article aims to expose the technological viability of a plant that produces a carob, tarwi, and quinoa drink through the definition of the product, the production process, and the plant capacity. This drink is an alternative to a large number of juices and sodas that have high sugar content. In addition, since it is made from a mixture of two legumes and a pseudo-cereal, it provides essential amino acids to the human body. KEYWORDS: tarwi / quinoa / carob / drink / technological feasibility / plant capacity

*Corrreo electrónico: maggie.manriquep@gmail.com; rociovalverdem18@gmail.com; Rvillan@ulima.edu.pe 


\section{INTRODUCCIÓN}

Actualmente, según un estudio realizado por Euromonitor (2019) sobre las tendencias de consumo de bebidas no alcohólicas, los peruanos son más conscientes de su salud y de las repercusiones de ingerir bebidas carbonatadas o con un alto contenido de azúcar. Además, este estudio menciona que el consumo de bebidas hechas a partir de plantas presentó un crecimiento de un 20 \% entre el 2014 y el 2019.

Según una encuesta realizada por la consultora Nielsen, un $35 \%$ de los peruanos practica una dieta que no es considerada alta en azúcar (Nielsen, 2016). Asimismo, la encuestadora Ipsos menciona que los sectores socioeconómicos A y B son las que más consumen este tipo de productos, en un $50 \%$ y $40 \%$ respectivamente (Ipsos Apoyo, 2009). Es por ello que en el presente artículo se presenta la alternativa de una bebida asociada con esta tendencia.

Una segunda tendencia del consumidor peruano es el incremento del consumo per cápita de granos andinos cuyo valor en el 2019 fue de 2,3 kilogramos y se espera que para el 2021 su consumo aumente hasta los 3,5 kilogramos por persona, según informó el Ministerio de Desarrollo Agrario y Riego (2019). Ello se debe al reconocimiento de las propiedades nutricionales que los caracterizan. De acuerdo con el estudio realizado por Cerezal et al. (2012), la formulación de mezclas de cereales y leguminosas permite obtener un mejoramiento del balance aminoacídico, lo que se traduce en un valor superior en la calidad de la proteína comparado con la de cada uno por separado.

Aprovechando dichas situaciones, se plantea ofrecer a los consumidores la alternativa de una bebida a base de quinua, tarwi y algarrobo con pulpa de fresa que no posee un alto contenido de azúcar. Por esto, el proyecto busca encontrar la tecnología apropiada para su manufactura, la cual debe adaptarse a los requerimientos de la demanda. De acuerdo con la segmentación de mercado del proyecto, se determinó que la cantidad demandada de botellas al año es de 799640.

Para exponer la viabilidad tecnológica del proyecto, se utilizaron diferentes estudios relacionados. Uno de ellos es Estudio para la instalación de una planta productora de bebida energética gasificada a base de maca negra, hoja de coca y arándano (Agramonte y Ronceros, 2016) que utiliza el método de filtro prensa para separar los sólidos de los líquidos. Otro de los artículos utilizados es Implementación de una planta de elaboración de bebida de papaya (Carica papaya) con linaza (Linum usitatissimum) (Urquizo-Baldarrago, 2015) que detalla el proceso de cocción en una marmita para obtener el gel de linaza. Del mismo modo, se utilizó el artículo La industrialización de una bebida natural a partir del tumbo andino (Passiflora mollissima) con linaza (Linum usitatissimum) (CórdovaLavado, 2016) como guía para los procesos de envasado y tapado semiautomático. Como referencia de un método de desinfección para los insumos de la bebida, se utilizó la 
desinfección con hipoclorito de sodio al igual que en el artículo Diseño y elaboración de bebida de aguaymanto (Physalis peruviana) enriquecida con kiwicha (Hernández et al., 2019)

\section{MÉTODO DE LA INVESTIGACIÓN}

La investigación de la viabilidad tecnológica de la implementación de una planta de producción de una bebida a base de lupino, algarrobo y quinua en el Perú se realizará de acuerdo con los siguientes aspectos:

\section{- Definición del producto}

Se calculó las proporciones de insumos en la bebida a través de la experimentación. El porcentaje de proteína diario cubierto por la bebida, por rango de edad, y la cantidad de aminoácidos esenciales presentes en la bebida se obtuvieron a través de datos recolectados de fuentes secundarias. Asimismo, se establecieron las características de la bebida a través del cuadro de especificaciones técnicas, a partir del cual se definieron los atributos del producto y su tipo, el nivel de criticidad, los medio de control, la frecuencia de control, la técnica de inspección y el nivel de calidad aceptable (NCA) de cada uno de ellos.

\section{- Definición del proceso de producción}

En el presente artículo, se presenta el diagrama de operaciones del proceso en el cual se muestran gráficamente los puntos en los cuales los materiales son introducidos en el proceso y la secuencia de inspecciones y de todas las operaciones.

\section{- Balance de materia y definición de la capacidad de la planta}

Se utilizó el método matemático de balance de materia con el fin de calcular la cantidad de insumos ingresantes y salientes en cada actividad del lote de producción. Para ello, se utilizó el tamaño de lote como la cantidad de producto terminado en el diagrama.

Para definir la capacidad de planta fue necesario hallar la operación cuello de botella. Se utilizaron los datos de cantidad entrante según balance de materia, la capacidad de procesamiento por hora, el número de máquinas en cada proceso, el factor de utilización y el factor de eficiencia.

Una vez definida la capacidad de planta, se elaboró un diagrama de actividades múltiples con el fin de determinar la cantidad de lotes de producción anuales que cubran la demanda del proyecto. Finalmente, validando que se cuenta con la tecnología requerida, se determina la viabilidad tecnológica del proyecto. 


\section{RESULTADOS}

El producto de la presente investigación es una bebida a base de dos leguminosas y un pseudocereal, que tiene la característica principal de aportar algunos aminoácidos esenciales que el organismo requiere.

Esta bebida es presentada en botellas de vidrio de 250 mililitros, con una tapa tipo twist-off y con una etiqueta que cumple lo establecido en la Norma Técnica de Etiquetado de Alimentos Envasados. Como ejemplo de ello, se encuentra la fecha de vencimiento, contenido neto, lista de ingredientes y registro sanitario (Indecopi, 2010). Además, cada botella de esta bebida aporta 3,4 gramos de proteína.

En la figura 1, se muestra el prototipo de la bebida a entregar al consumidor final.

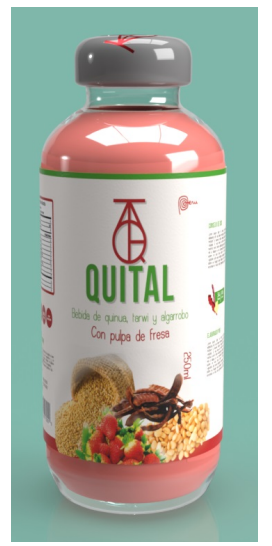

Figura 1. Prototipo de la bebida

Elaboración propia

La composición de la bebida a base de algarrobo, tarwi y quinua con pulpa de fresa se presenta en la tabla 1.

Tabla 1

Proporción de insumos de la bebida a base de lupino, algarrobo y quinua

\begin{tabular}{cc}
\hline Componente & Porcentaje \\
\hline Agua de tarwi & 40 \\
Agua de quinua & 21 \\
Agua de algarrobo & 15 \\
Pulpa de fresa & 20 \\
Azúcar & 3,93 \\
Ácido cítrico & 0,07 \\
Total & 100 \\
\hline
\end{tabular}

Elaboración propia 
El agua de tarwi se obtiene de una relación agua:grano de 6:1, el agua de quinua de 6:1 y el agua de algarrobo de 5:1 en el proceso de cocción (Cerezal et al., 2012), con la restricción de que la mezcla de estas aguas de cocción aporte del $75 \%$ al $80 \%$ de la formulación y que cumpla con la aprobación de la evaluación sensorial realizada.

Por otro lado, el mismo estudio establece que la bebida preparada tiene un contenido de proteínas de 1,36 \%. Según esta información, se puede calcular que el total final de proteínas en la bebida es de 3,4 gramos como lo muestra la tabla 2 .

Tabla 2

Gramos de proteína en la bebida

\begin{tabular}{ccc}
\hline $\begin{array}{c}\text { Porcentaje de proteínas } \\
\text { en la bebida }\end{array}$ & $\begin{array}{c}\text { Peso de la bebida } \\
(\mathrm{g})\end{array}$ & $\begin{array}{c}\text { gramo de proteínas } \\
\text { en la bebida }\end{array}$ \\
\hline 1,36 & 250 & 3,4 \\
\hline
\end{tabular}

Fuente: Cerezal et al. (2012)

Elaboración propia

De ese dato, se puede calcular el porcentaje de aporte diario que otorga la bebida según la edad del consumidor. En la tabla 3, se muestra un ejemplo aproximado del porcentaje diario de proteína cubierto por la bebida.

Tabla 3

Porcentaje de proteína diario cubierto por la bebida por rango de edad

\begin{tabular}{ccccc}
\hline Edad & $\begin{array}{c}\text { Peso de } \\
\text { ejemplo (kg) }\end{array}$ & $\begin{array}{c}\text { gramo de proteína/kg } \\
\text { de peso recomenda- } \\
\text { dos por día (FAO) }\end{array}$ & $\begin{array}{c}\text { gramo de proteína } \\
\text { recomendados por día }\end{array}$ & $\begin{array}{c}\text { Porcentaje de } \\
\text { proteína } \\
\text { diario cubierto } \\
\text { por la bebida }\end{array}$ \\
\hline $3-5$ & 16 & 1,5 & 24,0 & 14 \\
$5-16$ & 35 & 1,3 & 45,5 & 7 \\
$16 a+$ & 60 & 1,0 & 60,0 & 6 \\
\hline
\end{tabular}

Fuente: FAO (s. f.)

Elaboración propia

Asimismo, una de las características más relevantes de la bebida es su contenido de aminoácidos. Por ello, se presenta la cantidad de aminoácidos en gramos por cada 100 gramos de proteína contenidos en la bebida en la tabla 4. 
Tabla 4

Aminoácidos esenciales en la bebida

\begin{tabular}{lc}
\hline Aminoácidos esenciales & $\mathrm{g} / 100 \mathrm{~g}$ de proteína \\
\hline Isoleucina & 0,0272 \\
Leucina & 0,0492 \\
Lisina & 0,0300 \\
Metionina + cistina & 0,0178 \\
Fenilalanina + tirosina & 0,0529 \\
Treonina & 0,0308 \\
Triptófano & 0,0044 \\
Valina & 0,0283 \\
Histidina & 0,0115 \\
\hline
\end{tabular}

Fuente: Cerezal et al. (2012)

Igualmente, se presenta el cuadro de especificaciones organolépticas y físicoquímicas de la bebida.

Tabla 5

Especificaciones técnicas de la bebida

\begin{tabular}{|c|c|c|c|c|c|c|c|}
\hline $\begin{array}{l}\text { Característica } \\
\text { del producto }\end{array}$ & Tipo & $\begin{array}{l}\text { Nivel de } \\
\text { Criticidad }\end{array}$ & V.N. \pm Tol & $\begin{array}{l}\text { Medio de } \\
\text { control }\end{array}$ & Frecuencia & $\begin{array}{l}\text { Técnica de } \\
\text { Inspección }\end{array}$ & NCA \\
\hline $\begin{array}{l}\text { Sólidos } \\
\text { solubles }\end{array}$ & Variable & Crítica & ${ }^{\circ}$ Brix $>0,75$ & Refractómetro & Por lote & Muestreo & 1,5 \\
\hline $\mathrm{pH}$ & Variable & Crítica & $\mathrm{pH}<4,5$ & $\mathrm{pH}$-metro & Por lote & Muestreo & 1 \\
\hline Color & Atributo & Mayor & Rosado & Visual & Por lote & Muestreo & 1,5 \\
\hline Olor & Atributo & Mayor & $\begin{array}{c}\text { Libre de } \\
\text { olores } \\
\text { indeseables }\end{array}$ & Olfato & Por lote & Muestreo & 1,5 \\
\hline Sabor & Atributo & Mayor & Característico & Gusto & Por lote & Muestreo & 1,5 \\
\hline $\begin{array}{l}\text { Estado del } \\
\text { envase }\end{array}$ & Atributo & Mayor & Sin daños & Visual & Por lote & Muestreo & 1,5 \\
\hline
\end{tabular}

Elaboración propia

A continuación, se detalla el proceso de producción de la bebida de la presente investigación.

- Recepción de materias primas: El proceso inicia con la recepción de materias primas que son los sacos de los granos de quinua, tarwi y las vainas de algarrobo. También, se reciben las bolsas de pulpa concentrada de fresa y el ácido cítrico en diferentes presentaciones. 
- Desvainado de algarrobo: Se desvaina manualmente el algarrobo para separar las semillas, las cuales posteriormente serán lavadas y seleccionadas.

- Lavado y selección de materias primas: Seguidamente, los granos de tarwi y quinua y el algarrobo son lavados con agua con hipoclorito en lavaderos industriales usando coladores.

- Pesado: Se pesa las tres materias primas en balanzas de mesa según la cantidad a entrar por lote.

- Cocción: Las cocciones del tarwi, quinua y algarrobo se realizan en marmitas en proporciones de agua de 1 a 6, siendo la mayor proporción la del agua. Los tiempos de cocción son de 100, 23 y 38 minutos para el tarwi, quinua y algarrobo, respectivamente.

- Licuado: A continuación, se realiza el licuado de las aguas de cocción y las materias primas por un tiempo aproximado de 15 minutos.

- Filtrado: Después del licuado, con el uso de un filtro prensa que cuenta con un agujero de membrana de filtrado de 100 micras, se separan, casi en su totalidad, los sólidos de la mezcla, cuyo peso es de un $16 \%$ aproximadamente.

- Preparado de la fórmula (pulpa): A su vez, se prepara la formulación de pulpa de fresa adicionando el azúcar y el ácido cítrico. Esta mezcla se calienta a $80^{\circ} \mathrm{C}$ por un tiempo de 20 minutos.

- Mezclado: Posteriormente, se mezcla la cantidad filtrada con la pulpa preparada en un tanque con agitador por 10 minutos.

- Pasteurizado: Se realiza el pasteurizado de la mezcla a fin de producir un choque térmico que inhibe el crecimiento de microorganismos.

- Lavado de botellas: Para lavar las botellas, se coloca 12 botellas a la vez en la máquina enjuagadora y las botellas son trasladadas a la mesa de envasado.

- Envasado: Se envasa en botellas de $250 \mathrm{ml}$ con una envasadora de pistón de llenado que se ajusta al volumen requerido por botella.

- Tapado y etiquetado: Se tapa las botellas manualmente y se realiza el sellado con una tapadora twist-off y, luego, se etiqueta cada botella manualmente.

- Control de calidad: Se realiza un control de calidad del etiquetado en cuanto a la orientación de la etiqueta, validando que los bordes de esta no estén despegados. También se verifica que la superficie superior de la tapa no esté levantada por ser un tapado twist-off $\mathrm{y}$, visualmente, que el volumen de la bebida por botella sea el adecuado.

- Retractilado: Como último paso, un operario corta un metro de polietileno termoencogible con una cuchilla, coloca 12 botellas juntas encima de este, las 
envuelve $y$, con una pistola de calor, sopla la superficie del polietileno con el fin de realizar el retractilado.

A continuación, se muestra el diagrama de operaciones del proceso y el balance de materia prima respectivamente (figuras 2 y 3 ).

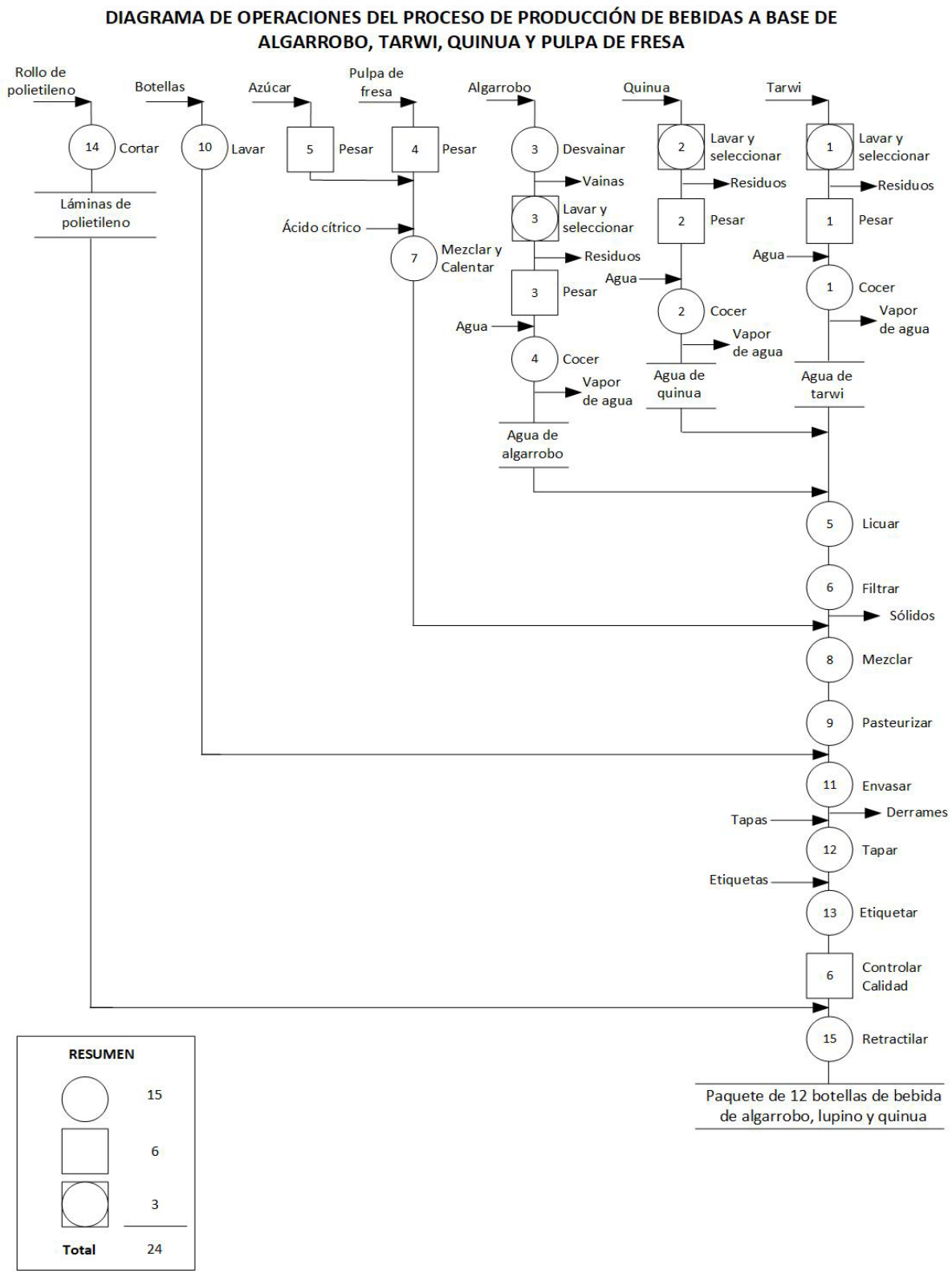

Figura 2. Proceso de producción de la bebida de algarrobo, tarwi y quinua Elaboración propia 


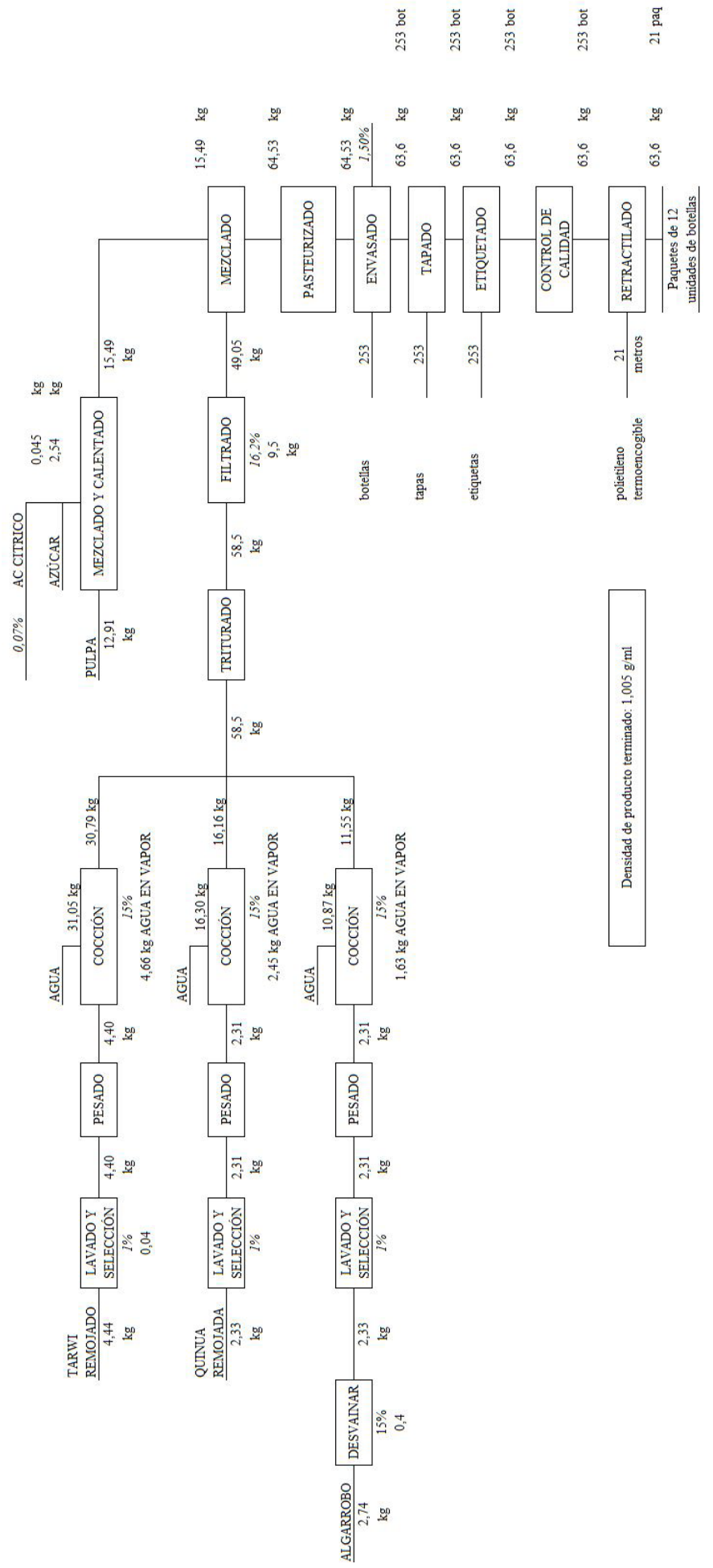

Ingeniería Industrial n. ${ }^{\circ}$ 41, diciembre 2021 


\section{En la tabla 6, se detalla la sustentación de la tecnología elegida para cada operación.}

Tabla 6

Tecnología seleccionada

\begin{tabular}{|c|c|c|}
\hline Operación & $\begin{array}{l}\text { Tecnología } \\
\text { elegida }\end{array}$ & Sustentación \\
\hline Desvainar & Manual & $\begin{array}{l}\text { Debido a la cantidad de materia prima por lote, se opta por realizar un } \\
\text { desvainado manual. }\end{array}$ \\
\hline Pesado & $\begin{array}{l}\text { Balanza de } \\
\text { mesa }\end{array}$ & $\begin{array}{l}\text { De acuerdo con las cantidades de materia prima por lote a pesar, se opta } \\
\text { por una balanza con un rango de pesaje de } 0 \text { a } 30 \mathrm{~kg} \text {. }\end{array}$ \\
\hline Lavado & $\begin{array}{l}\text { Lavado por } \\
\text { inmersión }\end{array}$ & $\begin{array}{l}\text { Considerando el tamaño de cada materia prima y que no es necesario } \\
\text { contar con una máquina especializada, el lavado será manual utilizando } \\
\text { coladores en estaciones de lavado. }\end{array}$ \\
\hline Cocción & Marmitas & $\begin{array}{l}\text { Como se busca conservar el mayor peso del líquido en la cocción, se opta } \\
\text { por cocinar en marmitas. Los tiempos de cocción son de 100, 22, } 38 \text { minu- } \\
\text { tos para el tarwi, la quinua y el algarrobo, respectivamente. }\end{array}$ \\
\hline Triturado & $\begin{array}{l}\text { Licuadora } \\
\text { industrial }\end{array}$ & $\begin{array}{l}\text { Para triturar y mezclar la materia prima con su respectiva cantidad de } \\
\text { agua de cocción, no es necesaria una máquina especializada. Por ello, se } \\
\text { utilizará una licuadora industrial con una capacidad de } 80 \text { litros, tomando } \\
\text { en cuenta la cantidad a licuar por lote. }\end{array}$ \\
\hline Filtrado & Filtro prensa & $\begin{array}{l}\text { Debido a que se busca separar los sólidos suspendidos, se opta por usar } \\
\text { un filtro prensa por el cual pasará el fluido y se retendrá aproximadamen- } \\
\text { te un } 16 \% \text { en peso del total de la mezcla. }\end{array}$ \\
\hline Mezclado & $\begin{array}{l}\text { Tanque con } \\
\text { agitador }\end{array}$ & $\begin{array}{l}\text { Debido a que no es necesario contar con una máquina especializada para } \\
\text { esta operación, se opta con un tanque con agitador, el cual cumple la fun- } \\
\text { ción de mezclar el fluido filtrado y la pulpa calentada previamente. }\end{array}$ \\
\hline Pasteurizado & $\begin{array}{l}\text { Pasteurizado } \\
\text { VAT }\end{array}$ & $\begin{array}{l}\text { Debido a que no es necesaria una pasteurización a una temperatura su- } \\
\text { mamente alta con el fin de que el tiempo sea de segundos, se opta por } \\
\text { una tecnología de pasteurizado de velocidad lenta. }\end{array}$ \\
\hline $\begin{array}{l}\text { Lavado de } \\
\text { botellas }\end{array}$ & Enjuagado & $\begin{array}{l}\text { Se escoge la tecnología del enjuagado debido a la cantidad de botellas a } \\
\text { lavar por lote. }\end{array}$ \\
\hline Envasado & $\begin{array}{l}\text { Envasado } \\
\text { con pistón de } \\
\text { llenado }\end{array}$ & $\begin{array}{l}\text { Se opta por el envasado con pistón de llenado ajustándolo a } 250 \mathrm{ml} \text { por } \\
\text { botella. }\end{array}$ \\
\hline Tapado & $\begin{array}{l}\text { Tapado al } \\
\text { vacío }\end{array}$ & $\begin{array}{l}\text { Con el fin de eliminar el aire dentro del frasco, se opta por un tapado al } \\
\text { vacío twist-off. }\end{array}$ \\
\hline Etiquetado & Manual & $\begin{array}{l}\text { Debido a la cantidad por etiquetar, el etiquetado será realizado manual- } \\
\text { mente con un tiempo de } 9 \text { segundos por botella. }\end{array}$ \\
\hline Retractilado & $\begin{array}{l}\text { Retractilado } \\
\text { con pistola } \\
\text { de calor }\end{array}$ & $\begin{array}{l}\text { Debido a que no es necesaria una máquina con una alta capacidad de pro- } \\
\text { ducción por hora, se opta por un retractilado manual con pistola de calor. }\end{array}$ \\
\hline $\begin{array}{l}\text { Mezclado y } \\
\text { calentado de } \\
\text { la pulpa }\end{array}$ & $\begin{array}{l}\text { Olla con } \\
\text { agitador }\end{array}$ & $\begin{array}{l}\text { No es necesaria alta tecnología para este proceso. Por ello, se utilizará } \\
\text { una olla de cobre con agitador eléctrico. }\end{array}$ \\
\hline
\end{tabular}

Elaboración propia 
Para determinar la capacidad instalada, se toma en cuenta que la producción de la elaboración de la bebida desarrollada en el proyecto es por lotes. Cada tiempo de ciclo es aproximadamente de 6 horas y media, obteniendo así un tamaño de lote de 253 botellas. También se toman en cuenta las capacidades de cada máquina seleccionada para la búsqueda de las opciones disponibles en el mercado.

Para el caso de las actividades manuales, se toma un factor de utilización de $100 \%$ y un factor de eficiencia de $83 \%$; este último porcentaje se calcula de acuerdo con los tiempos observados y estándares. En el caso de las operaciones que implican el uso de una máquina, se calculó un factor de eficiencia promedio de $88,8 \%$ de acuerdo con los tiempos de carga, descarga y de operación de cada máquina, así como el factor de utilización de 91 \%. En la tabla 7, se muestra el cálculo de número de máquinas.

Tabla 7

Cálculo de número de máquinas

\begin{tabular}{|c|c|c|c|c|c|c|c|}
\hline Máquina & $\begin{array}{l}\text { Producción de } \\
\text { máquina/ año } \\
\text { (kg) }\end{array}$ & $\begin{array}{c}\text { Capacidad } \\
\text { de la máquina } \\
(\mathrm{kg} / \mathrm{hr})\end{array}$ & Hrs/año & $U$ & $E$ & $\begin{array}{l}\mathrm{N} \\
\text { máquinas } \\
\text { teóricas }\end{array}$ & $\begin{array}{l}\mathrm{N} \\
\text { máquinas } \\
\text { reales }\end{array}$ \\
\hline Cocción del tarwi & 139162,74 & 26 & 6805 & 0,91 & 0,89 & 0,986 & 1,00 \\
\hline $\begin{array}{l}\text { Cocción de } \\
\text { quinua }\end{array}$ & 73060,44 & 80 & 1472 & 0,91 & 0,89 & 0,769 & 1,00 \\
\hline $\begin{array}{l}\text { Cocción de } \\
\text { algarrobo }\end{array}$ & 51728,93 & 33,6 & 2454 & 0,91 & 0,89 & 0,778 & 1,00 \\
\hline Triturado & 229669,32 & 375 & 1047 & 0,91 & 0,89 & 0,726 & 1,00 \\
\hline Filtrado & 201741,58 & 50 & 5366 & 0,91 & 0,89 & 0,933 & 1,00 \\
\hline $\begin{array}{l}\text { Mezclador de } \\
\text { bebida y pulpa }\end{array}$ & 253361,52 & 429 & 916 & 0,91 & 0,89 & 0,800 & 1,00 \\
\hline Pasteurizado & 253361,52 & 50 & 7198 & 0,91 & 0,89 & 0,873 & 1,00 \\
\hline $\begin{array}{l}\text { Mezclado y calen- } \\
\text { tado de pulpa }\end{array}$ & 60806,76 & 75 & 1309 & 0,91 & 0,89 & 0,768 & 1,00 \\
\hline $\begin{array}{l}\text { Lavado de } \\
\text { botellas }\end{array}$ & 799640 & 8640 & 3926 & 0,91 & 0,84 & 0,031 & 1,00 \\
\hline
\end{tabular}

Elaboración propia

En la tabla 8, se muestra el cálculo de los operarios requeridos para todas las operaciones considerando que un operario puede realizar más de una operación y tomando en cuenta el tiempo de ciclo. Además, se debe considerar que las operaciones no se crucen al realizar varios lotes. 
Tabla 8

Cálculo de número de operarios

\begin{tabular}{|c|c|c|c|c|c|c|}
\hline N & Operación & $\begin{array}{l}\text { Cantidad } \\
\text { entrante }\end{array}$ & Unidad & $\begin{array}{c}\mathrm{N} .^{\circ} \text { de } \\
\text { operarios }\end{array}$ & $\begin{array}{c}\text { Tiempo } \\
\text { (min) }\end{array}$ & $\begin{array}{c}\text { Tiempo } \\
\text { acumulado } \\
\text { (min) }\end{array}$ \\
\hline \multirow[t]{8}{*}{1} & Desvainar (algarrobo) & 2,74 & $\mathrm{Kg}$ & 1 & 16 & 16 \\
\hline & Lavado y selección algarrobo & 2,33 & $\mathrm{Kg}$ & & 4 & 20 \\
\hline & Pesado de algarrobo & 2,31 & $\mathrm{Kg}$ & & 2 & 22 \\
\hline & Cocción de algarrobo & 13,18 & $\mathrm{Kg}$ & & 38 & 60 \\
\hline & Lavado y selección quinua & 2,33 & $\mathrm{Kg}$ & & 4 & 64 \\
\hline & Pesado de quinua & 2,31 & $\mathrm{Kg}$ & & 2 & 66 \\
\hline & Cocción de quinua & 18,61 & $\mathrm{~kg}$ & & 22 & 88 \\
\hline & Retractilado & 253 & bot & & 15 & 103 \\
\hline 2 & Cocción del tarwi & 35,45 & $\mathrm{Kg}$ & 1 & 105 & 105 \\
\hline \multirow[t]{2}{*}{3} & Filtrado & 58,50 & $\mathrm{~kg}$ & 1 & 82 & 82 \\
\hline & Tapado & 253 & bot & & 28 & 110 \\
\hline \multirow[t]{2}{*}{4} & Mezclado y calentado de pulpa & 15,49 & $\mathrm{~kg}$ & 1 & 50 & 50 \\
\hline & Envasado & 253 & bot & & 50 & 100 \\
\hline 5 & Pasteurizado & 64,53 & $\mathrm{~kg}$ & 1 & 96 & 96 \\
\hline \multirow[t]{6}{*}{6} & Lavado y selección tarwi & 4,44 & $\mathrm{Kg}$ & 1 & 6 & 6 \\
\hline & Pesado de tarwi & 4,40 & $\mathrm{Kg}$ & & 2 & 8 \\
\hline & Triturado & 58,5 & $\mathrm{~kg}$ & & 16 & 24 \\
\hline & Lavado de botellas & 253 & bot & & 24 & 48 \\
\hline & Mezclador de bebida y pulpa & 64,53 & $\mathrm{~kg}$ & & 14 & 62 \\
\hline & Etiquetado & 253 & bot & & 34 & 96 \\
\hline
\end{tabular}

Elaboración propia

Luego de calcular el número de máquinas, se procedió a calcular la capacidad instalada considerando la siguiente fórmula:

Capacidad instalada $=\frac{k g}{\text { hora }} \times \frac{\text { horas }}{\text { turno }} \times \frac{\text { turno }}{\text { días }} \times \frac{\text { días }}{\text { año }} \times$ factores $\times$ número de máquinas

En la tabla 9, se muestra los cálculos para hallar la capacidad instalada que es delimitada por la operación cuello de botella, en este caso, la cocción del tarwi. 


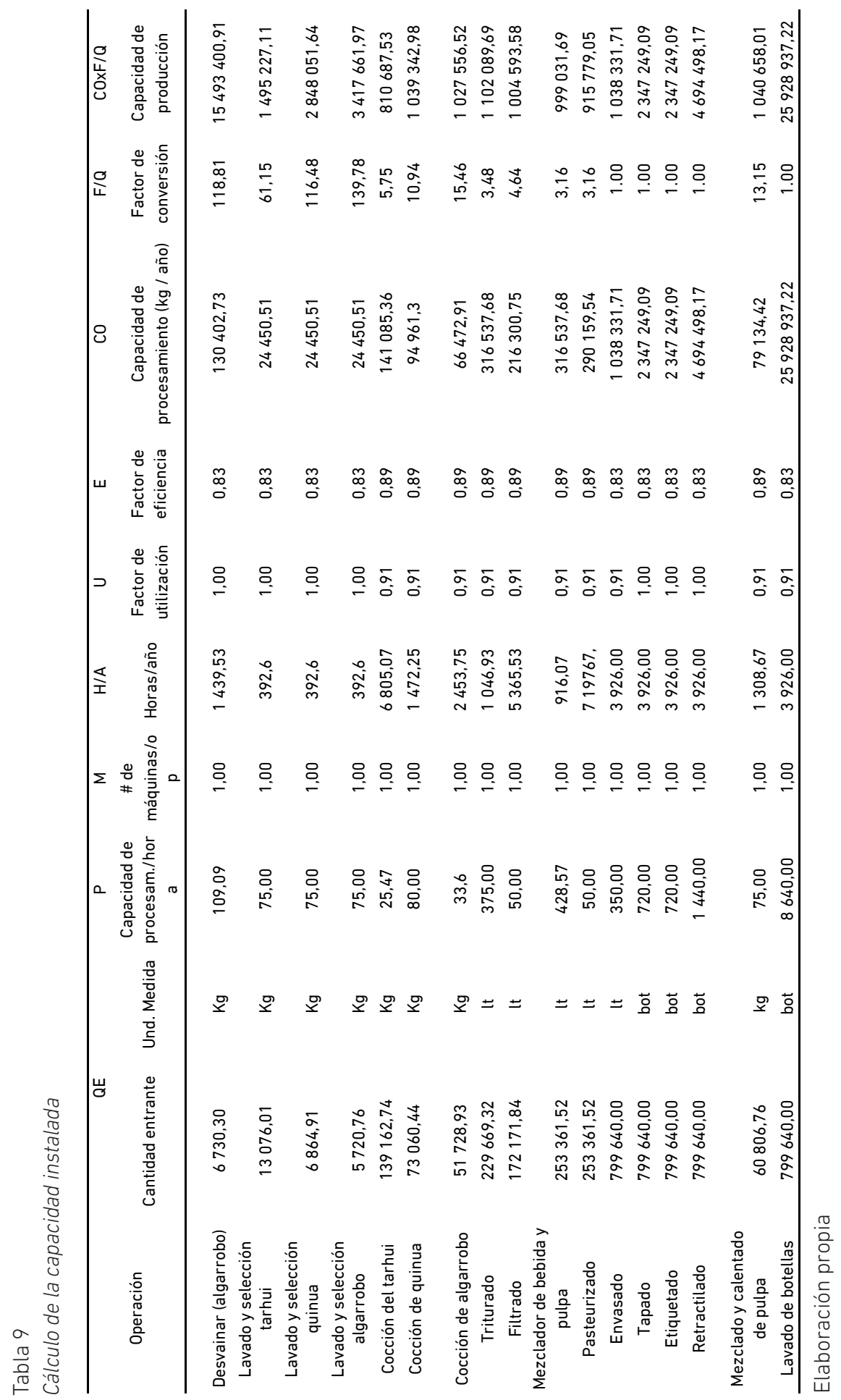


Se presenta, a continuación, el diagrama de actividades múltiples que se utilizó para obtener el tamaño de lote y el tiempo de ciclo del proceso. Con estos datos, se pudo calcular la capacidad instalada.

El tamaño de lote considerado es de $63,6 \mathrm{~kg}$ equivalente a 253 botellas y el tiempo de ciclo es de 6,4 horas. Gracias al diagrama y a los datos obtenidos, se pudo deducir que al día se elabora 13 lotes de producción y que cada 109 minutos se termina un lote.
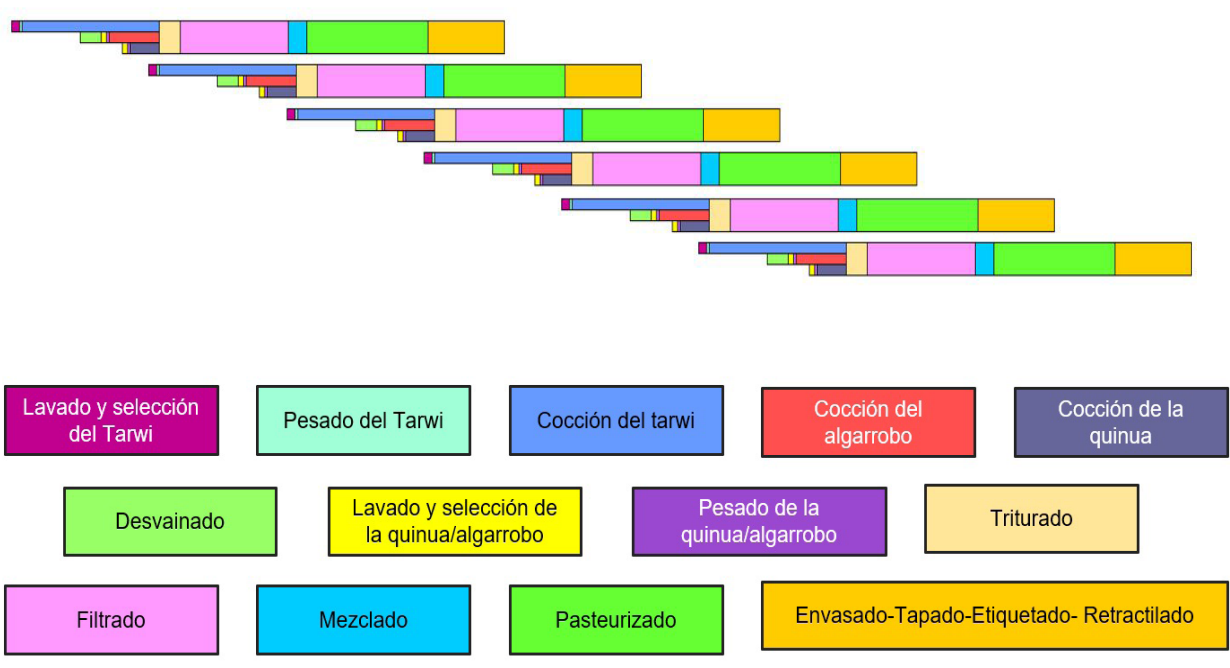

Figura 4. Diagrama de actividades múltiples

Elaboración propia

Luego de calcular el tamaño de lote, se determina la cantidad de materia prima que ingresará a cada actividad del proceso.

Finalmente, con los resultados obtenidos, se determina que la capacidad de la planta es de 810687 botellas al año, cubriendo así las 799640 botellas demandadas. En consecuencia, se demuestra la viabilidad de la tecnología seleccionada.

\section{CONCLUSIONES Y RECOMENDACIONES}

Según lo analizado en el presente trabajo de investigación, se puede concluir y recomendar lo siguiente:

- Los factores clave para el cálculo de la capacidad de planta fueron la segmentación del mercado y la determinación de la demanda anual. 
- Para la selección de tecnología, se definió que algunos procesos sean manuales o semiautomáticos, considerando que existe tecnología que supera la capacidad de procesamiento necesaria para estas actividades.

- La identificación de la operación cuello de botella permitió determinar la cantidad de lotes de producción diaria.

- Es necesario controlar todos los parámetros (cantidad de insumos, tiempo y temperatura) para obtener los niveles de proteínas y aminoácidos propuestos, ya que todos estos influyen directamente en el producto.

- De acuerdo con lo expuesto, se valida que la instalación de una planta productora de bebida de algarrobo, tarwi y quinua es tecnológicamente viable.

- Se recomienda realizar un estudio para evaluar si la torta procedente del proceso de filtrado puede reprocesarse para crear un nuevo producto terminado, siempre y cuando se asegure el cumplimiento de los estándares de calidad del cuadro de especificaciones técnicas.

\section{REFERENCIAS}

Agramonte Mendiola, D., y Ronceros Mac Kay, L. (2016). Estudio para la instalación de una planta productora de bebida energética gasificada a base de maca negra, hoja de coca y arándano. Ingeniería Industrial (34), 177-194. https://revistas.ulima.edu. pe/index.php/Ingenieria_industrial/article/view/1345

Cerezal, P., Acosta, E., Rojas, G., Romero, N., y Arcos, R. (2012). Desarrollo de una bebida de alto contenido proteico a partir de algarrobo, lupino y quinoa para la dieta de preescolares. Nutrición Hospitalaria, 27(1), 232-243.

Córdova-Lavado, I. C. (2016). La industrialización de una bebida natural a partir del tumbo andino (Passiflora mollissima) con linaza (Linum usitatissimum). Ingeniería Industrial (34), 195-219. https://doi.org/10.26439/ing.ind2016.n034.1344

Euromonitor, (2019). Jugos en Perú. http://www.portal.euromonitor.com.ezproxy.ulima. edu.pe/portal/analysis/tab

FAO (s. f.). Necesidades nutricionales. http://www.fao.org/docrep/014/am401s/am401s 03.pdf

Hernández, E., Vergara, J., Carlos, N., Inostroza, L., García, M., Villafuerte, U., Córdova, J., Birne, R., Alencastre, A., Peña, M., y Tupiño, O. (2019). Diseño y elaboración de bebida de Aguaymanto (Physalis peruviana) enriquecida con kiwicha. Ciencia e Investigación, 22(1):35-39

IPSOS Apoyo. (2009). Tendencias en salud y alimentación 2008. 
Ministerio de Desarrollo Agrario y Riego. (2019). Consumo de granos andinos llega a 2,3 kilogramos per cápita anual. https://www.gob.pe/institucion/midagri/noticias /45213-consumo-de-granos-andinos-llega-a-2-3-kilogramos-per-capita-anual

Nielsen. (5 de octubre del 2016). El $49 \%$ de los peruanos sigue dietas bajas en grasa, ubicándose en el segundo lugar de latinoamérica. https://www.nielsen.com/pe/ es/insights/news/2016/El-49-por-ciento-de-los-peruanos-sigue-dietas-bajasen-grasa.html

NTP 209.038:2009, Alimentos Envasado. Etiquetado. (20 de febrero del 2010). Indecopi.

Urquizo-Baldarrago, K. N. (2015). Implementación de una planta de elaboración de bebida de papaya (Carica papaya) con linaza (Linum usitatissimum). Ingeniería Industrial, (33), 181-203. https://doi.org/10.26439/ing.ind2015.n033.541 